statistics I have gathered from my own experience. It is a very small proportion in which the nose plays any role at all. Two cases were quoted which seem to be open to serious doubt unless supported by much more detail than the brief synopsis given. One was ulcer of the cornea. I do not think it has ever been noticed by anyone before that ulcers of the cornea are in any way kept up by intranasal conditions. Indirectly an intranasal disease may lead to ulceration of the cornea, the infection may be conveyed through the fingers to the eyelids and conjunctival sac, or in some cases the infection spreads from the nose to the sac, leading to dacryocystitis. In either case the infection of the cornea having been started, the remote cause no longer has any influence over it. I have not seen anything suggested by actual experience which would show that nasal treatment would influence the course of corneal ulceration. Another instance was quoted, namely, color blindness having been relieved by intranasal treatment. Color blindness, as far as we can judge, is purely a psychic phenomenon. No one has demonstrated that there is a any anomaly of the eye. It is not an acquired thing. Outside of disease of the optic nerve it is a psychic occurrence, and how that could be induced by intranasal anomalies has not been shown by the experience of anyone else. Undoubt. edly there are quite a large number of important troubles of the eye which owe their origin to the nose. To those which he has mentioned I might add two varieties. One is the production of iritis in consequence of intranasal suppuration. My attention was called to it by Ziehme, who claimed to have seen it produced by antral suppuration. I have the records of four patients with recurrent attacks of iritis, most of the attacks being distinctly preceded by purulent rhinitis, limited to one side, and the corresponding side of the nose was struc. turally anomalous. There was either narrowness or deviation of the septum or some of the other conditions known to influ. ence unfavorably the course of nasal suppuration. A second observation is that in some instances peripheral paralyses of the ocular muscles are due to intranasal conditions. I have in a previous paper referred to three successive cases that had come under my observation. I have seen two others since, where the paralysis occurred in one of the branches of the third nerve or was limited to the abducens, where the entire course and recovery characterized the trouble as peripheral. In all these patients the paralysis was preceded by acute sup. puration in the side of the nose corresponding to the af fected eye.

Dr. William L. Ballenger.-The essayist mentioned enlargements of the sphenoid and ethmoid bones, and I am not sure whether he meant simply hypertrophy of the bony tissue or a dilatation of the cells. But what $I$ want to refer to is suppurative diseases of the accessory cavities. Let us take, for instance, empyema of the sphenoidal sinuses. If you will recall the anatomy of the parts, the position in which the optic nerve passes through the foramen and on to the globe of the eye, you will see that it passes over the sphenoidal sinus, and if there is an empyema of any considerable extent of the sinus the bony tissue becomes inflamed, softens, yields and is pressed upward, and as it presses upward the optic nerve is choked or compressed at its entrance into the foramen and we may have either a sudden, partial or complete blindness as a result. If we have empyema of the ethmoidal cells, pressure inward will produce disturbance of the muscles of the eye, and strabismus of one type or another may result. There is another condition related to suppurative disease of the ethmoidal cells which resembles dacrocystitis very much. A little lump appears upon the skin, but not in the same situation as in dacrocystitis, but above the inner canthus of the eye. This is due to a pouching of the fluids or pus formation in the ethmoidal cells.

I have never seen a case of polypus of the inferior turbinated body reported. Zuckerkandl, in his great work, says he has never found polypus of the inferior turbinated body. I think it is generally accepted as a clinical fact that polypi do not occur here. I think that most of the inflammatory diseases of the conjunctiva and lachrymal apparatus, which the Doctor mentioned, would not necessarily be extensions from the nasal tract, but that the condition which caused the nasal disease also caused the eye disturbance. We do not understand very fully the nature of these inflammatory conditions, especially of the acute type. It is reasonable, however, to believe that the conditions have.a common origin in something that has not been mentioned.

Dr. HAIGHT. - In answer to Dr. Gradle's remarks about the condition of the nose having nothing to do with a persistent ulcer of the cornea, I will say that 1 had, in my clinic at the Chicago Eye, Ear, Nose and Throat College, a boy 12 yeare old, who had a phlyctenular ulcer of the cornea lasting one year.
This boy had been treated in one of the post-graduate schools, in this city, nine months previous to the time he came to me, three months ago. He had much photophobia, prof use lachrymation, the upper lid was swollen so that the eye could not be opened by patient. There was watery discharge from the nose with post-nasal adenoids and enlarged tonsils. The adenoids and tonsils were removed and the nose treated in conjunction with the eye, and now the eye is entirely well with the exception of this resulting corneal opacity, which is clearing up. In connection with the case of color blindness I refer to the cases reported by Hamilton. While the case I reported of complete color blindness is the only one that came under my notice, still I have had other cases of contraction of the visual fields. What causes it I do not know. Dr. Ballenger misunderstood me. In my paper I spoke of polypoid degeneration of the inferior turbinated body, a condition which we frequently find.

\section{TRAUMATIC CORNEAL ULCER; REPORT OF A CASE.}

BY J. W. SHERER, M.D. KANSAS CITY, MO.

Nov. 9, 1897, N. O., male, age 55, Swede laborer, applied at the clinic of Professor W. C. Tyree of the Kansas City Medical College, for dispensary treatment for an injury of the left eye.

Previous history.-No ocular disease had been experienced. Hard chancre was reported to have been contracted twenty years ago but none of the usual syphilitic sequelæ followed. General health has always been robust and an open air life led. Of late years there has been some inconvenience from advancing presbypia, but the aid of glasses has not been sought.

Present condition.-November 6, while crushing stone with a hammer a fragment, presumably of stone, struck the left eyeball and caused intense pain. Rubbing and wiping the eye, an effort was made to continue the work. Next day the eye was still painful, became red and some discharge was observed. The symptoms increased in severity, causing sleeplessness, loss of appetite, perpetual shooting pains through the head, violent pain in the eyeball and painful photophobia.

Examination.-November 9 , the fourth day of the diserse: $\mathrm{O} . \mathrm{D} . \mathrm{V} .=5 / \mathrm{x} . \mathrm{O} . \mathrm{S} . \mathrm{V} .=2 / \mathrm{L}$. The vision secured with the right eye was practically normal for the light and card used; the exterior of this eye was also normal. Ocular tension normal. In the left eye there was severe bulbar conjunctivitis, most intense in the ciliary zone, and some injection of the blepharal conjunctiva. The lids were somewhat swollen, edematous and heavy. In the culs-de-sac some mucopus was found, but no microscopic examination was made. A shallow, central corneal ulcer was found which was foul and progressive. Its diameter was about three millimeters, its edges undermined and a narrow boundary of cloudiness marked the limit of cellular infiltration. The remainder of the cornea was clear. The iris was slightly injected, its motility sluggish. Pupil three millimeters. Ocular tension normal. Ophthalmoscopic examination of the right eye showed clear media, normal fundus and hypermetropia $=+.50$. In the left eye the fundus could not be readily seen but the reflex was normal and, obliquely, the media appeared clear and fundus normal.

With $a+16$, the ulcer was seen to be an excavation with overhanging edges. It contained much débris, slough and pus. A diagnosis was made of infected corneal ulcer from traumatic contusion and laceration and treatment ordered as follows: Hot compresses four times daily twenty minutes at a time, the com- 
presses to be kejt constantly hot by plunging them in hot water; atropin sulphate 1 per cent. aqueous solution, 1 drop applied three times daily; free use of warm, sterile saturated solution of boric acid four times daily; a lump of 5 per cent. aristol ointment to be put in the eye each eve. The patient was requested to keep the eye bandaged and to report at the clinic three times a week.

November 11, patient reported improvement; "slept for first time in several days;" pain intermits and is lighter; pupil dilated to five millimeters only; swelling reduced Treatment was continued and calomel laxative added. November 14, some chemosis present; pain is severe at times. Patient has not worked since second day of the injury. The ulcer is spreading downward slightly. With a small Bowman's lens scoop and free irrigation with boric acid solution, the ulcer was curetted. The edges were much undermined. Remem. bering the patient's suspicion of specific taint I put him on potassium iodid 0.65 gram three times daily.

The patient attended the clinic regularly and reported that all directions were carried out, but his home was badly ventilated and warmed and his assistance was poor. After curettement some improvement was observed; epithelium proliferating over the upper part of the ulcer. November 20 there was an acute exacerbation with development of much chemosis. The ulcer was again foul and curettement was repeated.

When next seen the patient reported much improvement under the treatment. A little later the favorable progress of the case was again interrupted. The denudation of corneal epithelium had extended inferiorly. The iris was discolored and remained undilated. Intense ocular and blepharal conjunctivitis was present with sufficient swelling of the membrane to cause some eversion of the lower lid. Some pus was precipitated in the anterior chamber. The lids were rubbed with silver nitrate, 1 per cent. solution, and the ulcer wiped out with a wisp of cotton dipped in the same solution. A little later this was repeated with improvement, the hypopyon being almost completely absorbed. Then another relapse occurred, the ulcer spreading downward and a heavy hypopyon forming. The whole lower segment of the cornea was cloudy and was apparently becoming infiltrated with pus. November 20 the patient came into the hospital and paracentesis was performed by Professor Tyree. With antiseptic precautions and under cocain, section was made with a Graefe knife through a very tough cornea, in its lower segment, and a mass of pus sufficient to fill one-third of the anterior chamber was discharged on the cheek. After irrigation and applying aseptic dressings the patient was sent to the ward. The complete antiphlogistic and antiseptic treatment was now executed by skilled hands.

At this juncture elixir of quinin, iron and strychnin was ordered in place of the iodid. 'ine patient's strength was somewhat impaired by the suffering and nervous strain. He now suffered less and felt better, but soon the entire cornea rapidly became opaque, the lower half yellow and apparently infiltrated with pus. The chemosis again became great; the conjunctivitis was general and increased in severity and the swelling of the lids became worse. Within a few days the lower half of the cornea became markedly staphylomatous; the loss of the eye seemed at hand. The treatment was carefully continued with the addition of applications of silver nitrate, 1 per cent. solution, to the blepharal conjunctiva every other day, and the exhibition of a calomel laxative, pro re nata.

The patient suffered but little from the time the paracentesis was done, and December 5 the general inflammatory condition of the eye and tutamina was perceptibly improved. Henceforth the improvement was continnous. The patient slept well, had a voracious appetite, little pain, and gained in weight. December 21 , he was discharged from the hospital, all inflammation having subsided, with the cornea normal in contour and clear, except in the lower half, where a faint white, cloudy leucoma marked the area of the more severe keratitis. A few fine blood vessels had proliferated into this part of the cornea. At the time of writing, Jan. 7, 1898, O. S. V. $=3 / 50$; the cornea is slightly facetted; the leucoma bids fair to clear up partly, either spontaneously or under stimulative treatment; the pupil is regular; the iris normal in motion and color; there seems to be no disturbance of the ocular metabolism, and the lids are healthy.

The points of interest are: 1 , the extremely bad irritative symptoms caused by the movement of the lids over the wound and which were relieved by immo. bilization with a bandage; 2 , the ulcer extended down in the line of gravitation of the pus in the anterior chamber and to the area of the cornea not covered by the lids; 3 , the change in course of the disease with paracentesis, hospital and tonic treatment; 4 , the utter failure of the antiseptic and antiphlogistic treatment at the patient's home under pernicious sanitary and hygienic environment and in unskilled hands; $\tilde{0}$, the fortunate issue of the case from grave danger; 6 , the disappearance of large quantities of pus from the anterior chamber by filtration through the mesh-work of the ligamentum pectinatum. 7 , the certain loss of the eye had paracentesis not been done and had the patient not been kept in hospital.

310-11 New Ridge Building.

\section{A REPORT OF A CASE OF ANOMALOUS THORACIC ANEURYSM.}

Read before the Chicago Medical Soclety, Feb. 9, 1898. BY G. W. JOHNSON, B.Sc., M.D.

Medical Superintendent of the Cook County Infirmary; Late Chief Male Physician to the Cook County Hospital for the Insane; Fellow of the American Academy of Medicine; Member of the American

Medical Association, Chicago Medical Society, and the Mississippi Valley Medical Association. CHICAGO, ILL.

Aneurysm of the aorta is not an uncommon occur. rence in the experience of every physician, and from the group of symptoms found in text-books on practice and surgery is not especially difficult of diagnosis. That there are exceptional cases, however, in which a diagnosis is somewhat difficult, there is little doubt. Such a case is the one herein reported.

After having studied this case for some months and then being unable to make a satisfactory diagnosis, I presented the case to the Chicago Medical Society for diagnosis. A number of its most able clinicians became interested, and after considerable study and repeated examinations agreed upon a diagnosis of lymphosarcoma of the mediastinum, the tumor occupying the right thorax. The clinical and personal history, together with postmortem notes and the author's deductions, are here given in full:

Personal history.-W. D., aged 53 years, Irish, unmarried, is a plumber by occupation, venereal history negative, no history of alcoholism, typhoid fever, pneumonia or pleurisy. Has 